\title{
Influence of periodic wall roughness on the slip behaviour at liquid/solid interfaces: molecular-scale simulations versus continuum predictions
}

\author{
By NIKOLAI V. PRIEZJEV† AND SANDRA M. TROIAN \\ School of Engineering and Applied Science, Princeton University, Princeton, NJ 05844, USA
}

(Received 27 July 2005 and in revised form 11 January 2006)

The influence of surface roughness on the slip behaviour of a Newtonian liquid in steady planar shear is investigated using three different approaches, namely Stokes flow calculations, molecular dynamics (MD) simulations and a statistical mechanical model for the friction coefficient between a corrugated wall and the first liquid layer. These approaches are used to probe the behaviour of the slip length as a function of the slope parameter $k a=2 \pi a / \lambda$, where $a$ and $\lambda$ represent the amplitude and wavelength characterizing the periodic corrugation of the bounding surface. The molecular and continuum approaches both confirm a monotonic decay in the slip length with increasing $k a$ but the rate of decay as well as the magnitude of the slip length obtained from the Stokes flow solutions exceed the MD predictions as the wall feature sizes approach the liquid molecular dimensions. In the limit of molecularscale wall corrugation, a Green-Kubo analysis based on the fluctuation-dissipation theorem accurately reproduces the MD results for the behaviour of the slip length as a function of $a$. In combination, these three approaches provide a detailed picture of the influence of periodic roughness on the slip length which spans multiple length scales ranging from molecular to macroscopic dimensions.

\section{Introduction}

The possibility of liquid slippage along the surface of a solid and conditions favouring this behaviour have been debated for over two centuries. Many historical figures including Bernoulli, Coulomb, Navier, Couette, Poisson, Stokes, Poiseuille, Hagen and Helmholtz devoted significant effort in identifying the proper hydrodynamic boundary condition at the surface of contact between a liquid and solid in relative motion (Goldstein 1938). By the 19th century, three distinct views had emerged, namely $(a)$ that the tangential speed of the liquid equals that of the adjacent solid surface (i.e. the no-slip condition), (b) that the liquid slips against a thin film immobilized by wall imperfections, and (c) that the liquid slips against the solid surface with a tangential speed proportional to the viscosity and shear rate, as first proposed by Navier.

The no-slip condition was the most widely accepted at the time given its success in reproducing the velocity profiles for macroscopic flows. By 1938, the Fluid Motion

$\dagger$ Current address: Department of Mechanical Engineering, Michigan State University, East Lansing, MI 48824, USA 


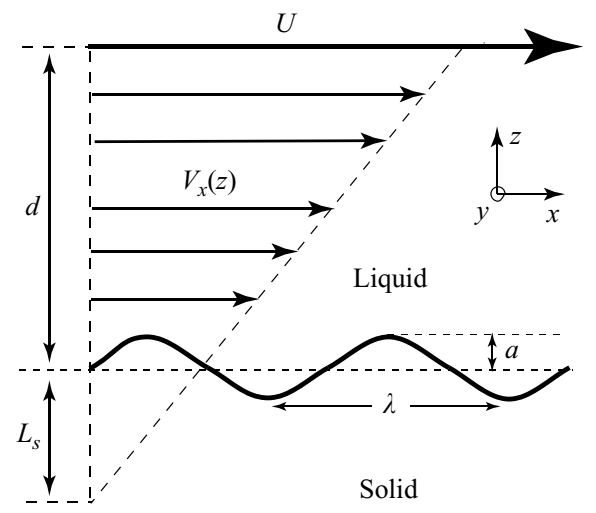

FIGURE 1. Plane Couette cell geometry used for the MD simulations and continuum calculations. The surface of the stationary lower wall is represented by a sinusoid with maximum amplitude $a$ and wavelength $\lambda$. The wall separation distance for $a=0$ is designated $d$. The upper flat wall moves at a constant speed $U=1.0 \sigma / \tau$ in the MD simulations. The slip length $L_{s}$ is computed by linear extrapolation of the velocity field to the value $V_{x}=0$.

Panel of the Aeronautical Research Committee, writing in a landmark two-volume series (Goldstein 1938) devoted to important developments in boundary layers, turbulent flow and wakes, concluded that even if the Navier slip condition were correct, the slip length was likely to be only a moderate multiple of the liquid molecular mean free path and therefore too small to influence the behaviour of macroscopic flows. Recent developments in microfluidic and nanofluidic devices have renewed interest in the problem of slip, where its influence is expected to be significant even as the Reynolds number $R e \rightarrow 0$.

In postulating the slip boundary condition, Navier invoked a similar basis to that which forms the foundation of the equations of motion for a viscous fluid. He argued that in the presence of slip, the liquid motion must be opposed by a force proportional to the relative velocity between the first liquid layer and the solid wall (here assumed stationary). Reasoning that the tangential stress is continuous across this interface, he concluded that

$$
\beta V_{s}=\mu \partial V / \partial n
$$

where $V_{s}$ is the tangential liquid speed at the solid surface, $n$ is the unit normal pointing away from the solid, $\partial V / \partial n$ is the liquid shear rate at the solid surface, $\mu$ is the liquid viscosity and $\beta$ is the friction coefficient. The ratio $\mu / \beta$, which he assumed constant and independent of the shear rate, is nowadays designated the slip length $L_{s}$. For the Couette geometry shown in figure 1 where the lower wavy wall is stationary and the upper flat wall translates at a constant speed $U$, the slip length is obtained by linear extrapolation of the tangential velocity component to zero velocity within the solid phase. In investigating the spreading of cells and viscous droplets on a planar solid substrate, Greenspan (1978) invoked a similar slip condition for free-surface flows where $L_{s}$ is replaced by the ratio $\alpha / 3 h$, where $\alpha$ is the slip coefficient and $h(x, y)$ the local liquid film thickness. This alternative condition allows for increased slip in regions of high shear rate. Both slip conditions eliminate the stress singularity in corner flows (Moffatt 1964; Huh \& Scriven 1971).

There remains to this day significant debate concerning the validity of slip conditions as well as the degree of slip possible at liquid/solid interfaces. Beyond the form originally proposed in (1.1), there is now interest in determining what 
molecular-level parameters govern the degree of slip and whether the slip length $L_{s}$ depends on the local shear rate. During the past decade, experimentalists have used optical and rheological techniques of increasing resolution to deduce estimates of the slip length. Among these are evanescent-wave-induced fluorescence combined with photobleaching (Migler, Hervet \& Leger 1993; Pit, Hervet \& Leger 2000; Schmatko, Hervet \& Leger 2005), microparticle image velocity (Tretheway \& Meinhart 2002; Jin et al. 2004), precision flow metering (Choi, Westin \& Breuer 2003), fluorescence cross-correlation of labelled tracer particles (Lumma et al. 2003) and the surface force apparatus (Horn et al. 2000; Baudry et al. 2001; Zhu \& Granick 2002; Cottin-Bizonne et al. 2005), which confines liquid films to gap sizes of the order of nanometres. Given some lingering discrepancies among the results of different groups, uncertainties in the composition of the liquid film adjacent to a solid wall (which influences the extracted value of the slip length), and recent reports of spontaneous nucleation of gas nanobubbles at water/hydrophobic interfaces (Ishida et al. 2000; Tyrrell \& Attard 2001; Steitz et al. 2003), various questions involving the slip length remain unsolved. The increasingly popular field of micro- and nanofluidics has also introduced some important practical considerations, such as what are the best liquids or surface treatments to use in order to maximize slip and therefore reduce frictional losses while minimizing device size and input power. Answers to these questions require a much better understanding of the molecular origin of slip in the presence of ubiquitous substrate roughness.

\subsection{Analytical studies of liquid slip}

In addition to practical considerations related to drag reduction at low $R e$, there are certain fundamental hydrodynamic problems which require a modification of the no-slip boundary condition in order to avoid spurious flow singularities. For example, Moffatt (1964) derived a number of similarity solutions for the velocity fields arising in corner flows. He examined the flow of a viscous liquid near a sharp corner between two flat planes (either two rigid or one rigid and one free liquid surface) and determined that the no-slip boundary condition causes an $r^{-1}$ divergence in the shear stress, where $r=0$ is the corner point. Huh \& Scriven (1971) and Dussan \& Davis (1974) noted this is a non-integrable singularity which also arises in corner flows describing the region near a moving contact line, as in a liquid droplet spreading on a solid substrate. This singularity can be eliminated by replacing the no-slip boundary condition with a slip condition (Dussan V. 1979).

The idea first proposed by Girard (Goldstein 1938) that a liquid undergoes slip against a thin layer immobilized by surface imperfections directed attention toward the influence of wall roughness. Hocking (1976) examined the behaviour of a fluid interface separating two immiscible fluids flowing across a corrugated surface defined by a periodic array of parallel grooves. For sufficiently deep corrugations where one liquid is trapped in the hollows of the wall corrugation, he showed that the second liquid will essentially slide against the liquid-filled grooves with a finite shear stress. He estimated that the effective slip length in such a two-fluid system is proportional to the hollow depth if the corrugation is shallow, and to the hollow separation distance if the corrugation is deep. Richardson (1973) considered whether the no-slip condition is just an inevitable consequence of fluid trapping by surface roughness. He derived the inner Stokes solution corresponding to the flow of a liquid against a periodically undulated substrate representing a boundary of no-slip or no-shear (i.e. perfect slip). Surprisingly, the no-slip condition emerges as the relevant macroscopic boundary condition in both cases. Deviations from the no-slip condition enter at the same order 
of magnitude as the characteristic size of the wall asperities (assumed to be much larger than the liquid molecular size). Jansons (1988) examined the flow of a liquid near a wall containing a random array of microscopic defects occupying a small area fraction. In agreement with Richardson (1973), he found that a local slip condition along the surface of a roughened boundary leads to an effective no-slip condition at macroscopic length scales. Miksis \& Davis (1994) reconsidered this configuration in the limit of small-amplitude but arbitrary wall roughness and derived in systematic fashion the appropriate boundary conditions for flow over a roughened dry or liquidwetted surface. For single-phase liquid flow, the slip coefficient appearing in the Navier condition was shown to be linearly proportional to the average amplitude of the wall roughness. For a second fluid completely wetting the roughened wall, the slip coefficient depends on the viscosity ratio of the two liquids and the dynamic behaviour of the liquid-liquid interface through the relevant capillary number. Tuck \& Kouzoubov (1995) extended this analysis by including inertial corrections for low-Reynolds-number flow past a solid boundary of arbitrary roughness. Use of the no-slip condition along the wavy boundary leads to a backflow near the wall, whose profile can be equivalently obtained by applying a slip boundary condition along the plane defined by the geometric mean surface. Along the mean surface plane, Tuck \& Kouzoubov (1995) showed that the slip length is linearly proportional to the wavenumber corresponding to the weighted mean of surface roughness and quadratically proportional to the amplitude corresponding to the RMS value of surface roughness. Einzel, Panzer \& Liu (1990) and Panzer, Liu \& Einzel (1992) derived an analytic expression for the slip length which explicitly includes microscopic slip as well as the effect of local wall curvature (representative of mesoscopic surface roughness). The details of this model will be presented in $\S 4.1$ since the results of this hydrodynamic analysis form the basis of comparison to the molecular dynamics studies reported here.

\subsection{Molecular dynamics studies of liquid slip}

During the past decade molecular dynamics (MD) simulations have emerged as a powerful tool for probing the microscopic behaviour of liquids in bulk and near interfaces (Koplik \& Banavar 1995). Numerous studies have shown how the interaction potential and molecular packing or entropy constraints near a solid substrate influence both in-plane and out-of-plane ordering in the adjacent liquid molecules. The degree of in-plane ordering is characterized by the peak value of the liquid structure factor describing the molecular ordering in planes parallel to a flat wall. Out-of-plane ordering is manifested by multiple density oscillations that gradually decay toward the average value of the liquid density in the bulk. In general, the higher the degree of substrate-induced liquid ordering near a flat smooth wall, the smaller the degree of slip and the larger the momentum transfer between the liquid and solid. The molecular picture which has emerged, based on simplified statistical mechanical models (Bocquet \& Barrat 1994; Barrat \& Bocquet 1999a, $b$; Priezjev \& Troian 2004; Priezjev, Darhuber \& Troian 2005) and MD simulations (Thompson \& Robbins 1990a,b; Thompson, Brinckerhoff \& Robbins 1993; Thompson, Robbins \& Grest 1995; Koplik \& Banavar 1995; Gao, Luedtke \& Landman 1997; Jabbarzadeh, Atkinson \& Tanner 2000; Gao, Luedtke \& Landman 2000) (and many references therein) indicates that the slip length is strongly influenced by the strength of the liquid/solid interaction potential and the degree of commensurability between the liquid molecules and wall atoms. This commensurability is quantified by the amplitude 
of density oscillations near the wall as well as the in-plane structure factor and diffusion coefficient within adjacent layers. The no-slip condition emerges as one limit of a number of allowable boundary conditions ranging from perfect slip (i.e. infinite slip length) to epitaxial locking of one or two liquid layers to the solid wall (i.e. negative slip length). For the flow of two immiscible fluids across the surface of a solid (which includes the well-studied problem of a droplet spreading on a smooth solid substrate), MD simulations have shown just how sensitive the spreading dynamics and motion of the contact line are to the molecular nature of the liquid/solid interface (Thompson \& Robbins 1989; Thompson et al. 1993). Recent MD studies of simple and polymeric fluids subject to steady planar shear also reveal an interesting powerlaw dependence of the slip length on shear rate even at small Reynolds number (Thompson \& Troian 1997; Priezjev \& Troian 2004).

The influence of surface roughness on liquid flow has only recently been investigated by MD simulations. Jabbarzadeh et al. (2000) explored the behaviour of hexadecane and shorter alkane molecules subject to planar shear between two sinusoidally roughened walls. Long-chain molecules generate larger slip velocities with increasing sinusoidal period (i.e. smoother surfaces) and smaller slip velocities with increasing sinusoidal amplitude (i.e. rougher surfaces). The fluid viscosity and normal pressure were also found to decrease slightly with increasing wall period. Gao et al. (2000) investigated the flow behaviour of hexadecane against two uncorrelated rough surfaces prepared in the simulations by melting and then rapidly cooling a 10-layer FCC molecular stack with an exposed (110) surface. An interfacial fluid layer was found to adhere to the roughened wall which essentially recovers the no-slip condition. Subsequent liquid layers exhibited partial slip over the adhered layer. It was noted that molecular-scale roughness induces frustration in the molecular ordering near the solid. Galea \& Attard (2004) modelled surface roughness by varying the size and spacing of the wall atoms while maintaining a constant packing fraction. The density oscillation amplitude increases for smoother substrates, since more efficient packing of liquid molecules can occur within each layer adjacent to the wall. The no-slip condition was recovered in the limit where the wall atom size and spacing are commensurate with those in the liquid. In general, larger slip lengths are possible along smoother and locally compact walls since the likelihood of liquid trapping by crevices and bumps is significantly reduced.

In a recent study (Priezjev et al. 2005), we examined the flow of a Lennard-Jones (LJ) fluid subject to planar shear in a Couette cell. The bottom stationary surface was patterned by a periodic array of stripes representing alternating regions of finite slip (or no-slip) and no-shear (i.e. perfect slip). These mixed boundary conditions provide a first-order approximation to a periodic array of gas nanobubbles pinned against a solid wall. Random arrays of such nanobubbles have recently been observed in experiments (Ishida et al. 2000; Tyrrell \& Attard 2001; Steitz et al. 2003). The different boundary conditions were achieved by adjusting the magnitude of the attractive term in the LJ interaction potential used in the MD simulations which controls the degree of liquid affinity for the solid wall. Direct comparison of the numerical results from a Stokes flow analysis to the MD simulations showed excellent agreement between the velocity profiles and the slip length for stripe periods exceeding approximately 10-20 molecular diameters for flow configurations either parallel or transverse to the stripe pattern. For smaller stripe periods, the transverse flow configuration generated slip lengths below the hydrodynamic predictions. This behaviour was attributed to an effective molecular-scale roughness corresponding to the heterogeneity in liquid affinity along the solid surface. 
The focus of the current work is a systematic investigation of the effects of topological roughness for the system geometry shown in figure 1 . We investigate the behaviour of the slip length with increasing slope parameter $k a$ using three approaches, namely MD simulations, continuum calculations and a statistical mechanical model which describes the friction coefficient between a wavy wall and the first liquid layer. In the MD simulations, the wavy wall is constructed by sinusoidal displacement of wall atoms comprising an FCC lattice. This modulation induces a variation in wall atom spacing as seen by the fluid, an effect which in itself modifies the slip length. Corrections incorporating this effect are properly accounted for in making comparisons to various continuum models. In this study, the parameters characterizing the wall/fluid interactions were chosen to reproduce partially wetting boundary conditions. In the absence of any imposed wall corrugation, the intrinsic slip length, designated throughout by the parameter $L_{o}$, is comparable with the wall separation distance $d$. The parameters in the molecular model were specifically chosen to yield an appreciable degree of slip in order to provide a sufficient range for study since the surface roughness causes a rapid decay in slip length with increasing $k a$.

Implementing the MD simulations described in $\S 2$, we first investigate the velocity profiles for planar shear flow against a wavy wall by independently varying the corrugation amplitude and wavelength, as discussed in $\S 3$. We then review the asymptotic analytic result of Einzel et al. (1990) and Panzer et al. (1992) for the effective slip length obtained for pressure-driven (Stokes) flow bounded by two well-separated and wavy walls. This analysis highlights the effect of local wall curvature (or equivalently periodic surface roughness) in modulating the degree of slip. In $\S 4$, direct comparison is made between the MD results and continuum solutions in the Stokes regime for plane Poiseuille flow (Einzel et al. 1990; Panzer et al. 1992) and plane Couette flow. Good agreement is obtained in the limit of small-amplitude and large-wavelength corrugations. Improved agreement is obtained when the slip length substituted into the Einzel et al. relation is corrected for the variable wall atom spacing induced in the MD simulations by sinusoidal displacement of the wall molecules. Progressive reduction in the corrugation wavelength causes a suppression of the slip length with respect to the continuum predictions. To gain further insight into the behaviour of the liquid near the wall for molecular-scale corrugations, we extend the model by Barrat \& Bocquet $(1999 a, b)$ in $\$ 5$ by proposing a wall potential consisting of two competing wavelengths, one emerging from the wall lattice and the other from the imposed topological wall corrugation. This analysis is based on a Green-Kubo derivation of the friction coefficient between a wavy solid wall and the first adjacent layer of liquid molecules. In the limit where $\lambda$ approaches molecular-scale dimensions, the MD simulations exactly reproduce the analytic result for the behaviour of the slip length with increasing corrugation amplitude.

\section{Details of the molecular simulation method}

Interactions between fluid $(f)$ particles separated by a distance $r$ were modelled by a Lennard-Jones (LJ) potential

$$
V_{L J}(r)=4 \epsilon\left[\left(\frac{\sigma}{r}\right)^{12}-\left(\frac{\sigma}{r}\right)^{6}\right],
$$

whose minimum value $\epsilon$ corresponds to a particle separation distance $2^{1 / 6} \sigma$. Wall (w) and fluid particles also interact through an LJ potential with parameters $\epsilon_{w f}=0.7 \epsilon$ and $\sigma_{w f}=\sigma$. The interaction potentials were truncated at a cutoff radius $r_{c}=2.2 \sigma$; studies 
using a larger truncation radius $r_{c}=2.5 \sigma$ generated very similar velocity profiles. The smaller cutoff radius was therefore used in order to speed the computations (Thompson \& Troian 1997).

The simulation cell consisted of two parallel wall lattices each containing 756 particles bounding an interstitial slab of 5040 fluid particles. Each wall consisted of two layers whose atomic sites define the (111) plane of a face-centred cubic (FCC) lattice with density $\rho_{w}=3.38 \rho_{f}$, where $\rho_{f}=0.81 \sigma^{-3}$. The direction of shear, oriented along the $\hat{x}$-axis, was defined by the [11ㄹ] vector of the FCC lattice. The wall separation distance (see figure 1) was held fixed at a value $d=30.47 \sigma$. The surface area of the horizontal walls measured $L_{x} \times L_{y}=29.20 \sigma \times 7.22 \sigma$; periodic boundary conditions were enforced along the $\hat{x}$ - and $\hat{y}$-axes. The equations of motion governing the coordinate and momentum values of the $i$ th particle were integrated using a standard fifth-order Gear predictor-corrector scheme (Allen \& Tildesley 1987) with a time step $\Delta t=0.005 \tau$, where $\tau=\sqrt{m \sigma^{2} / \epsilon}$ represents the characteristic time scale of a Lennard-Jones fluid with particle mass $m$. The equation of motion for the acceleration along the $\hat{y}$-axis is given by

$$
m \ddot{y}_{i}=-\sum_{i \neq j} \frac{\partial V_{L J}}{\partial y_{i}}-m \Gamma \frac{\mathrm{d} y_{i}}{\mathrm{~d} t}+F_{i}(t) .
$$

Verlet lists were used to reduce the computational effort in calculating interatomic forces (Allen \& Tildesley 1987). The last two terms on the right, which are absent from the equations of motion for the $x$ - and $z$-components of the force on particle $i$, ensure isothermal fluid conditions by weakly coupling the particle dynamics to a Langevin thermal reservoir (van Gunsteren, Berendsen \& Rullmann 1981; Grest \& Kremer 1986; Thompson \& Robbins 1989, 1990 b; Thompson et al. 1993; Stevens et al. 1997). The friction coefficient $\Gamma$ regulates the heat flux from the system and $F_{i}$ represents a Gaussian random force with zero mean and variance $2 m \Gamma k_{B} T \delta(t)$ where $T$ is the fluid temperature, $\delta(t)$ is the delta function, and $k_{B}$ is Boltzmann's constant. In this study, $T=1.1 \epsilon / k_{B}$ and $\Gamma=1.0 \tau^{-1}$. This value of $\Gamma$ is large enough to remove heat from the system without inducing substantial changes in temperature but small enough to minimize disturbances to the particle trajectories. It has previously been shown for similar parameter values that the fluid temperature remains constant provided the fluid shear rate $\dot{\gamma}<U / d \lesssim 0.16 \tau^{-1}$ (Thompson \& Robbins $1990 b$ ). In our studies, the shear rate in the fluid never exceeded $0.033 \tau^{-1}$. Such isothermal control has been shown to be effective by just including the damping and Langevin noise terms in the $\hat{y}$-component of the equation of motion, i.e. the direction perpendicular to the direction of shear (Thompson \& Robbins 1989; Thompson et al. 1993; Stevens et al. 1997; Thompson \& Troian 1997). The fluid density and temperature used here represent a well-defined liquid phase at a temperature approximately $30 \%$ above the melting point (Heyes 1998). The validity of MD simulations as a probative tool was first established with reference to liquid argon for which $\sigma=3.4 \AA, \epsilon / k_{B}=120 \mathrm{~K}$, $m=6.69 \times 10^{-23} \mathrm{~g}$, and $\tau=2.161 \times 10^{-12} \mathrm{~s}$. A typical time step $\Delta t=0.005 \tau$ therefore corresponds to about $10^{-14} \mathrm{~s}$ which yields total run times in the microsecond range (see below).

The fluid was subjected to steady planar shear by translating the upper flat wall at a constant speed $U=1.0 \sigma / \tau$ along the $\hat{x}$-axis. The lower wavy wall remained stationary. The wavy wall was constructed by vertically displacing all particles within the (111)-plane of the FCC lattice by a distance $\Delta z=a \sin (2 \pi x / \lambda)$, where $a$ denotes the displacement amplitude and $\lambda$ the displacement wavelength, as designated in 


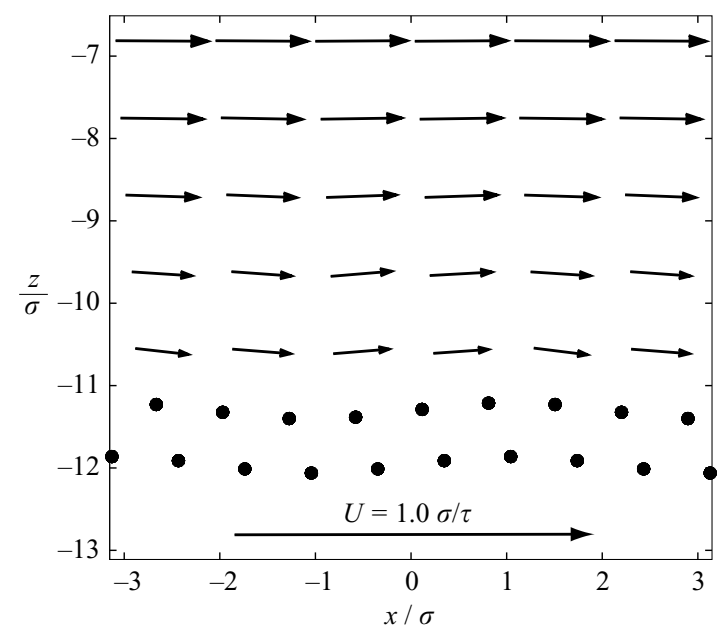

FIGURE 2. Velocity field vectors in the vicinity of the corrugated wall. The vectors represent the average particle velocities in the $(x, z)$-plane as computed from MD simulations for $a=0.1 \sigma, \lambda=4.18 \sigma$ and $d=22 \sigma$. The remaining parameters for the MD simulations are specified in the text. Black dots signify the centres of mass of the lower wall particles. The vector shown at the bottom of the figure represents the velocity $U=1.0 \sigma / \tau$ of the upper flat wall. $V_{x}(z)$ is essentially unidirectional beyond a vertical distance of a few particle diameters (about $2 \sigma-3 \sigma$ ) from the wall.

figure 1. This surface corrugation represents a periodic roughening of the lower wall boundary. For the MD simulations, the plane defined by $z=0$ was located at the mid-plane of the fluid phase. In this study, the corrugation wavelength spanned the range $1.82 \sigma \leqslant \lambda \leqslant 29.2 \sigma$ for $0 \leqslant k a \leqslant 1$; the corresponding corrugation amplitude was in the range $0 \leqslant a \leqslant 4.65 \sigma$. After an equilibration period of $\Delta t=2 \times 10^{4} \tau$, the steady-state velocity field $V_{x}(z)$ was reconstructed by averaging the particle speeds within horizontal slices $\Delta z=0.7 \sigma$ for a time interval $\Delta t \approx 2 \times 10^{5} \tau$. The simulations were performed on a 64 processor Linux Beowulf cluster. A typical run for computing velocity profiles required approximately 400 hours of CPU time. The Reynolds number based on an upper wall speed $U=1.0 \sigma / \tau$, a wall separation $d=30.47 \sigma$ and a fluid shear viscosity $\left(\mu=2.2 \pm 0.2 \epsilon \tau / \sigma^{3}\right.$ (Thompson \& Troian 1997; Priezjev \& Troian 2004) was estimated to be $R e \lesssim 10$. This estimate represents an upper bound not actually achieved within the fluid phase since slip at the walls reduces the internal shear rate. These simulation parameters generated an intrinsic slip length $10.0 \pm 0.3 \sigma$ for flow bounded by two flat wall (i.e. $a=0$ ). In what follows, this intrinsic slip length is designated $L_{o}$; the effective slip lengths obtained in the presence of wall corrugation are instead designated $L_{\text {eff }}$ in the continuum analysis and $L_{s}^{G K}$ in the statistical mechanical model.

\section{Velocity profiles for a viscous fluid in planar shear against a wavy wall}

The simulations described in $\S 2$ were used to compute the velocity profiles as a function of the slope parameter $\mathrm{ka}$. Shown in figure 2 is a representative projection in the $(\hat{x}, \hat{z})$-plane of the average particle velocities close to a wavy wall for $a=0.1 \sigma$ and $\lambda=4.18 \sigma$. The black dots indicate the centre-of-mass positions of the wall particles. The velocities were obtained by averaging the particle speeds within a narrow $x-z$ bin measuring $1.0 \sigma \times 0.5 \sigma$. For the small-amplitude distortions examined, the velocity fields retained a linear profile characteristic of plane Couette flow except within a 


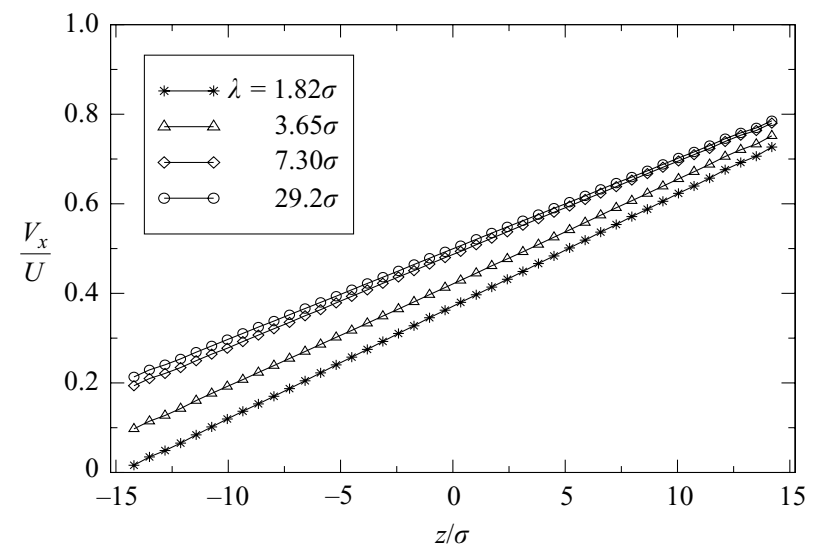

FIGURE 3. Velocity profiles $V_{x}(z)$ for $U=1.0 \sigma / \tau$ computed from MD simulations for increasing wall wavelength $\lambda$ at fixed amplitude $a=0.1 \sigma$. Owing to the small corrugation amplitude, the profiles are linear throughout, as expected for steady plane Couette flow. The slip velocity at the upper and lower walls increases with increasing wavelength. The choice $\lambda=1.82 \sigma$ essentially reproduces the no-slip condition at the lower wavy wall (left vertical axis) despite a finite slip velocity at the upper flat wall (right vertical axis).

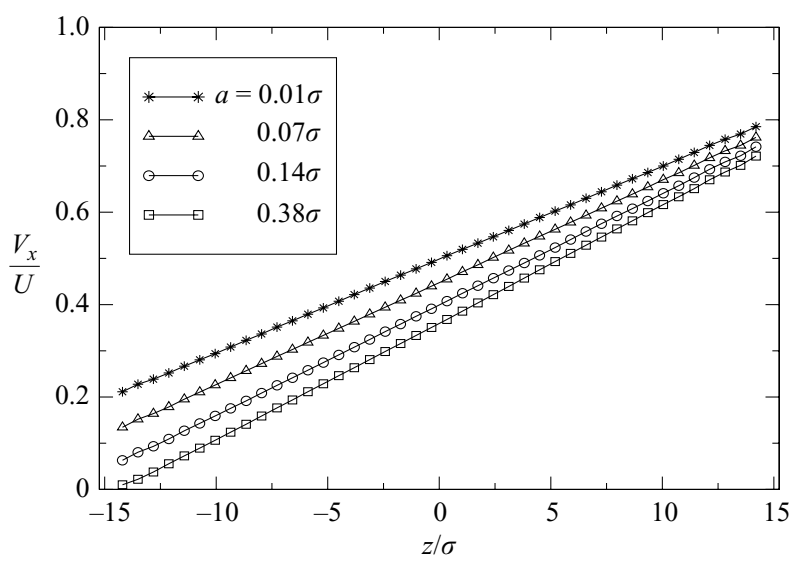

FIGURE 4. Velocity profiles $V_{x}(z)$ for $U=1.0 \sigma / \tau$ computed from MD simulations for increasing wall amplitude $a$ at fixed wavelength $\lambda=3.65 \sigma$. The slip velocity at the upper and lower walls decreases with increasing corrugation amplitude. The choice $a=0.38 \sigma$ reproduces the no-slip condition at the lower wavy wall (left vertical axis) despite a finite slip velocity at the upper flat wall (right vertical axis).

few molecular diameters $(2 \sigma-3 \sigma)$ of the wall boundary. Shown in figure 3 are representative velocity profiles $V_{x}(z)$ for increasing corrugation wavelength at fixed amplitude $a=0.1 \sigma$. The left vertical axis corresponds to the mean position of the bottom wavy wall and the right vertical axis to the position of the upper flat wall. Figure 4 demonstrates similar behaviour as a function of increasing corrugation amplitude at fixed wavelength $\lambda=3.65 \sigma$. Figure 3 indicates that the slip velocity at the wavy wall decreases for smaller values of $\lambda$. As shown in figure 4, larger corrugation amplitudes also decrease the degree of slip. In fact, the parameter values $a=0.10 \sigma$ and $\lambda=1.82 \sigma$ generate a no-slip condition at the wavy wall despite finite slip at the upper wall. This indicates that a corrugation amplitude only $1 / 10$ the 
effective LJ particle diameter is sufficient to immobilize a thin fluid layer for small corrugation wavelengths. In these studies, it was found that a slope ratio $k a=0.3$ effectively reproduced a no-slip condition.

These results clearly show how the intrusion of the wall boundaries into the fluid slows the flow speed and diminishes the value of the slip length. Previous studies by Thompson \& Robbins (1990 b) have shown that fluid adhesion and even epitaxial locking of the fluid to the supporting lattice can also occur with flat walls for larger values of $\epsilon_{w f}$ (i.e. larger affinity between the fluid and wall particles) and smaller ratios of fluid to wall density. In general, the more attractive the interaction coupling between the fluid and solid particles and the more commensurate the length scales between the wall feature sizes and the molecular diameter of the fluid particles, the more effective is surface roughness in slowing or even trapping the fluid in the wall interstices.

In the next section we examine the functional behaviour of the slip length with increasing $\mathrm{ka}$. Results from the MD simulations are directly compared to Stokes flow solutions. For the molecular simulations, linear extrapolation of the velocity profiles beyond the bottom wall was used to estimate the corresponding slip length, as indicated by the schematic diagram in figure 1. Direct comparison between these two approaches sheds some light on how the granularity or molecular nature of the fluid causes systematic deviations from hydrodynamic predictions.

\section{Comparison of molecular dynamics and continuum solutions}

Koplik \& Banavar (1995) provide an extensive discussion of the methods of molecular dynamics simulations as applied to a variety of viscous flows and the future prospects of direct comparison with continuum-based predictions. They conclude that molecular simulations, which are strictly based on small fluid volume size and nanosecond time intervals, can nonetheless provide a successful description of fluid flow over a range of length scales from molecular to macroscopic dimensions. Our previous study of flow over a chemically patterned surface (Priezjev et al. 2005) and the current study for flow over a topologically corrugated substrate indicate good correspondence with hydrodynamic predictions provided the length scale associated with wall inhomogeneities is larger than about $10 \sigma-20 \sigma$.

\subsection{Plane Poiseuille flow with wavy walls: Stokes-flow solutions}

Einzel et al. (1990), Panzer et al. (1992) and Einzel \& Parpia (1997) investigated the hydrodynamic flow of quantum liquids in restricted geometries. Experimental measurements at the time seemed to suggest smaller slip lengths than those computed from the generalized Landau-Boltzmann equation for the motion of quasi-particles, the elementary excitations in quantum fluids. Hydrodynamic predictions for normal ${ }^{3} \mathrm{He}$ also seemed to overestimate the degree of slip in Couette cells whose surfaces were wetted by a thin layer of superfluid ${ }^{4} \mathrm{He}$, which provides a friction-free liquid coating. Panzer et al. (1992) explored the role of surface imperfections in reducing the values of the slip length. Within the Stokes approximation, they analysed pressure-driven flow of a Newtonian liquid confined by two wavy walls. They isolated the effects of local substrate curvature and derived an expression for the effective slip length as a function of $k a$. An asymptotic analytic solution was obtained in the limit $k a \lesssim 0.5$. To satisfy the continuum approximation, it was assumed that the local radius of curvature describing the wall roughness was much larger than the molecular mean free path of the liquid. 
For a two-dimensional Cartesian coordinate system with the origin situated at the wavy boundary, the Navier slip condition is generalized to

$$
\frac{1}{L_{s}(x)}=\frac{1}{L_{o}}-\frac{1}{R(x)},
$$

where $L_{o}$ denotes the intrinsic slip length for a flat wall and $R(x)$ represents the local radius of curvature within the fluid medium along the flow direction. The liquid curvature is defined as positive for flow within an indentation and negative for flow over a solid bump (Einzel et al. 1990; Panzer et al. 1992). In the limit where the radius of curvature characterizing the flow $R \gg L_{o}$, (4.1) reduces to the Navier slip condition corresponding to a flat surface. The symmetry condition $V_{x}(x, z)=V_{x}(x, d-z)$ was also used to simplify the analysis. Additional simplifications were obtained by assuming that the surface corrugation is spatially periodic and that the corresponding amplitude $a$ and wavelength $\lambda=2 \pi / k$ are much smaller than the wall separation distance $d$. Their analysis showed that the Fourier components $C_{n}$ for the contribution to the fluid pressure induced by the wall curvature decay exponentially with increasing distance $z$ from the bottom wall according to $C_{n} \sim \exp (-n k z)$. The perturbation pressure caused by the wall modulation therefore penetrates the fluid only a distance on the scale of the corrugation wavelength.

In the limit of small $k a$ (i.e. the corrugation amplitude is much smaller than the wavelength), Panzer et al. (1992) showed that

$$
\lim _{k L_{o} \rightarrow 0} k L_{\mathrm{eff}}=k L_{o}-k^{2} a^{2} w_{o}(k a), \quad \lim _{k L_{o} \rightarrow \infty} k L_{\mathrm{eff}}=\left(\frac{1}{k L_{o}}+\frac{k^{2} a^{2}}{w_{\infty}(k a)}\right)^{-1},
$$

where

$$
w_{o}(k a)=\frac{1-(k a)^{2} / 4+19(k a)^{4} / 64+O\left[(k a)^{6}\right]}{1+(k a)^{2}-(k a)^{4} / 2+O\left[(k a)^{6}\right]}
$$

and

$$
w_{\infty}(k a)=\frac{1-5(k a)^{2} / 4+61(k a)^{4} / 64+O\left[(k a)^{6}\right]}{1+(k a)^{2}-(k a)^{4} / 2+O\left[(k a)^{6}\right]} .
$$

The normalized value of the effective slip length, namely $k L_{\text {eff }}$, is bounded above by $w_{\infty} /(k a)^{2}$ and bounded below by $(k a)^{2} w_{o}$. As expected, the correction term to the effective slip length in the limit $k L_{o} \rightarrow 0$ is a negative quantity, which Panzer et al. (1992) noted is due to the reduction in the effective wall separation distance $d-$ $2 k a^{2}\left|w_{o}\right|$. They also showed that the asymptotic expansions in (4.2)-(4.4) reproduce the results of the full numerical solutions for $k a \lesssim 0.5$. In completing their analysis, they also provided an approximate analytic expression for $L_{\text {eff }}$ which smoothly interpolates between the upper and lower bounds of the effective slip length, namely

$$
L_{\mathrm{eff}}=\frac{L_{o} w_{\infty}(k a)-\left[k a^{2} w_{o}(k a)\right] /\left(1+2 k L_{o}\right)}{1+k^{3} a^{2} L_{o}} .
$$

\subsection{Planar shear flow near a wavy wall: MD simulations}

To compare our MD results with the analytic expression (4.5) for $k a \lesssim 0.5$, it was necessary to incorporate an additional correction to the intrinsic slip length $L_{o}$ to account for the variation in wall atom spacing caused by the substrate corrugation. As discussed in $\S 2$, all particles comprising the two-layer solid wall were vertically displaced from their original positions within a flat plane by a distance $\Delta z=a \sin (k x)$. 


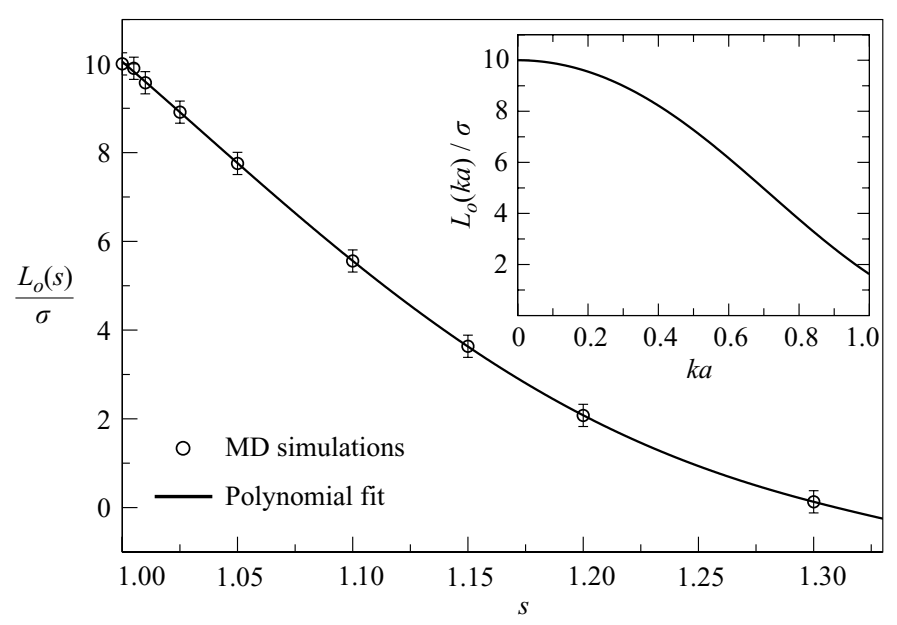

FIGURE 5. Results of MD simulations for the behaviour of the slip length with increased stretching parameter $s$, as defined in $\S 4.2$. The continuous line represents a fourth-order polynomial fit in the variable $p=s-1$, where $L_{o}(p)=10.0-44.8 p-38.3 p^{2}+429.7 p^{3}-572.1 p^{4}$. The inset shows the density-corrected slip length $L_{o}(s)$ re-plotted as a function of $k a$.

The subsequent variation in wall atom spacing along the corrugated surface naturally affects the interaction forces between the fluid and wall particles. In comparison to a flat wall with constant wall atom spacing and density $\rho_{w}$, the local wall particle density as seen by the fluid decreases in proportion to the boundary slope $\mathrm{d} h / \mathrm{d} x$. The steeper the local slope, the larger the inter-particle spacing and the smaller the effective local wall density. As described next, we incorporated this effect in an averaged way by introducing an overall stretching parameter. Previous MD studies of steady plane Couette flow bounded by flat walls (Thompson \& Robbins $1990 \mathrm{~b}$ ) indicate that a smaller ratio between the wall and liquid densities leads to a reduction in slip length since the fluid particles can be better accommodated within the interstices of the wall lattice particles. A similar effect is introduced by the variable wall spacing caused by the sinusoidal wall modulation.

To determine the approximate correction factor for $L_{o}$, we conducted a set of MD simulations for Couette flow confined by atomically flat walls where the spacing between wall particles was uniformly stretched by a factor $s$. This stretching parameter reduces the average wall density to $\rho_{w} / s$. To maintain a constant bulk fluid density, the wall separation distance $d$ was also decreased accordingly. Shown in figure 5 is a plot of the 'density-corrected' slip length $L_{o}(s)$ computed in this way. The continuous line overlying the data represents a fourth-order polynomial fit in the variable $p=s-1$. The inset figure shows the density-corrected slip length re-plotted as a function of $k a$. As expected, the slip length decreases monotonically with increasing $s$ since progressive stretching of the wall particle positions allows deeper embedding of the fluid particles within the wall matrix. In this study, the no-slip condition was reproduced for $s \approx 1.3$. Beyond this value, the effective slip achieves negative values indicating immobilization of the first fluid layer by the wall corrugations.

In order to account for the variable wall atom spacing induced by the corrugated boundary in the MD simulations, we used in the continuum analysis the data plotted in figure 5 but replaced the stretching parameter $s$ by the average value $\langle s\rangle=C / \lambda$, 

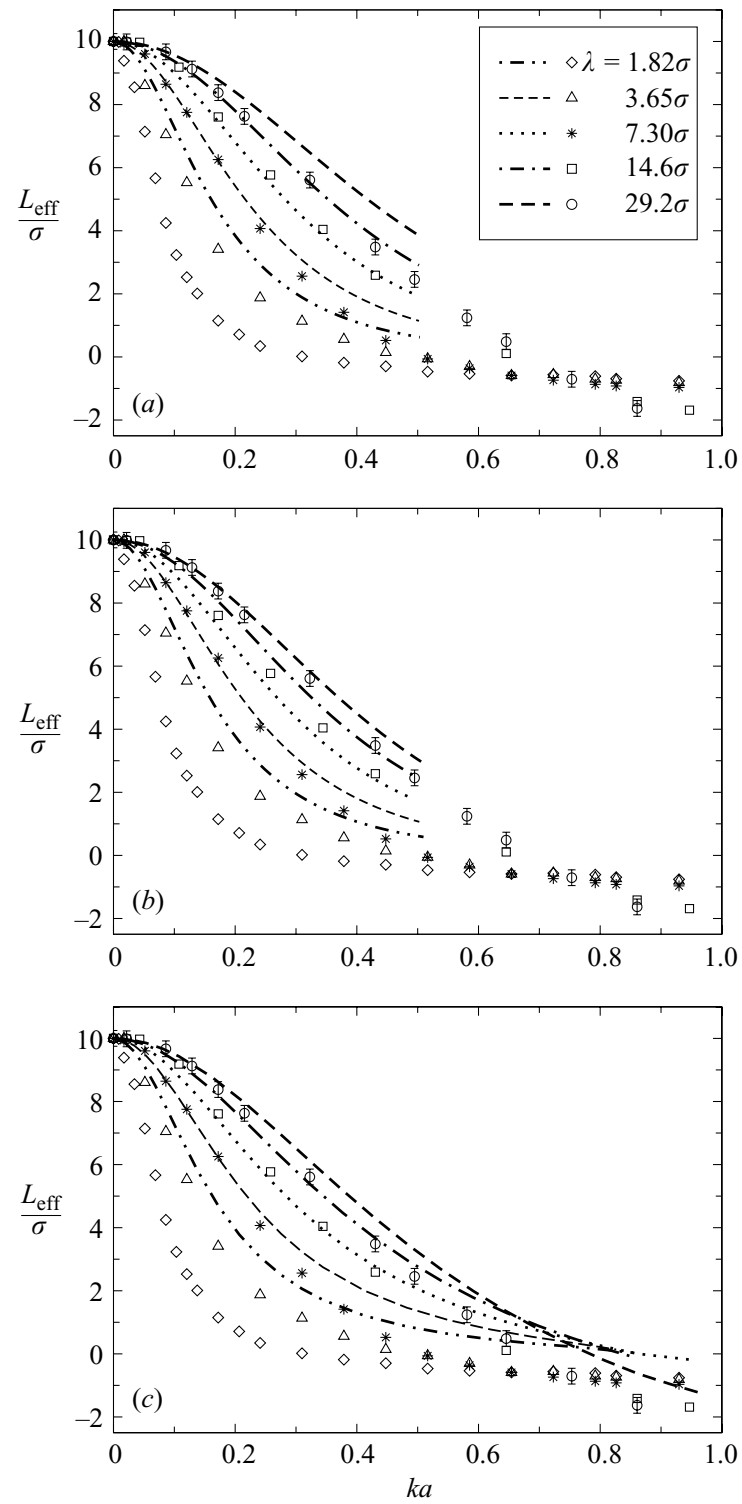

FiguRE 6. Comparison of MD results (open symbols-identical data in $(a-c)$ ) for the effective slip length as a function of slope parameter $k a$ with Stokes flow solutions (dashed lines) for three cases. (a) Dashed lines represent the asymptotic expression (4.5) where $L_{o}=10.0 \sigma$ for Poiseuille flow against wavy walls in the limit of large wall separation distance. $(b)$ Dashed lines represent the asymptotic expression (4.5) where $L_{o}$ has been replaced by the density-corrected slip length, $L_{o}(s)$, as specified by the polynomial curve in figure 5. (c) Dashed lines represent full numerical solutions for plane Couette flow over a single wavy wall subject to a constant slip length $L_{o}$ at the upper wall and a slope-dependent (i.e. local) slip length $L_{o}(s)$ at the bottom wall, as described by the polynomial curve in figure 5 .

where the contour length $C=\int_{0}^{\lambda}\left[1+(\mathrm{d} h / \mathrm{d} x)^{2}\right]^{1 / 2} \mathrm{~d} x=\int_{0}^{\lambda}\left[1+a^{2} k^{2} \cos ^{2}(k x)\right]^{1 / 2} \mathrm{~d} x$. Figure 6 represents a direct comparison of the MD results (the same MD data in (a)-(c)) with continuum predictions based on Stokes flow solutions for three different cases, namely two based on the analytical solutions for Poiseuille flow between two 
wavy walls derived by Einzel et al. and one based on numerical studies of Couette flow with a single stationary wavy wall, which corresponds exactly to the geometry used in the MD simulations. The dashed curves in figure 6(a) represent the solutions (4.5) (valid strictly for $k a \lesssim 0.5$ ) for $L_{o}=10.0 \sigma$. The dashed curves in figure $6(b)$ represent solutions where the slip length substituted into (4.5) has been corrected for the variable wall spacing by replacing $L_{o}$ by $L_{o}[\langle s\rangle]$ as discussed in figure 5 . The maximum value of the stretching parameter was $s=1.21$. This correction yields improved agreement between the MD and continuum predictions in the limit of small $k a$ and large $\lambda$.

The dashed curves in figure 6(c) represent numerical solutions in the Stokes limit for plane Couette flow in the presence of a single wavy wall whose surface is subject to a local slip condition based on the correction in figure 5. (The general solution procedure follows a derivation similar to that used previously for investigating plane Couette flow over a flat and chemically patterned surface (Priezjev et al. 2005).) The numerical solutions were obtained using the finite-element package FemLab 3.1 (COMSOL, Inc., Burlington, MA) with triangular elements and cubic basis functions. The wavy wall boundary was approximated by 400 line segments per half-period and the local slip length determined from the local boundary curvature. The slip length in the presence of a wavy wall was determined by comparison with numerical solutions for Couette flow between two flat walls separated by a distance $d$ subject to slip lengths $\ell_{\text {upper }}=L_{o}$ and $\ell_{\text {lower }}=L_{\text {eff }}$. The value of $\ell_{\text {lower }}$ was adjusted until the flow rate $Q$ obtained with flat walls matched the flow rate computed with one wavy wall. Since the velocity profile for Couette flow bounded by flat homogeneous walls is perfectly linear, the effective slip length follows from simple geometric considerations where

$$
\frac{L_{\mathrm{eff}}}{d}=\frac{\left(1+\ell_{\text {lower }} / d\right) A-1}{2-A} \text { and } A=\frac{2 Q}{U d} .
$$

Further details will appear in a future publication (Darhuber, Priezjev \& Troian 2006). The continuum solutions for plane Poiseuille and Couette flow in figures $6(b)$ and $6(c)$ in the limit of small $k a$ and large $\lambda$ (i.e. $\lambda=29.2 \sigma$ and $14.6 \sigma$ ) coincide almost exactly. This is probably because both velocity profiles in the vicinity of a slightly corrugated wall remain linear in $z$ (i.e Couette-like), as is the case near perfectly flat walls. As an aside, the continuum solutions shown in figure $6(a-c)$ were obtained in the Stokes limit where $R e=0$, while the MD simulations correspond to solutions for which $R e \lesssim 10$. Small inertial contributions in the MD simulations may be partly responsible for the small discrepancies observed between the MD and continuum results for low and moderate values of $k a$ and large $\lambda$.

For all three cases shown in figure $6(a-c)$, the effective slip length diminishes with increasing slope parameter $k a$. The smaller the corrugation wavelength $\lambda$, the more effective is the surface roughness in reducing the magnitude of the slip length. Good agreement between the various continuum solutions and the MD simulations is only obtained in the limit of small $k a$ and large $\lambda$ (i.e. $\lambda=29.2 \sigma$ and $14.6 \sigma$ ). For small but fixed values of $k a$, the correspondence worsens as $\lambda$ approaches the molecular length scale $\sigma$. In this limit, the fluid particles, characterized by an effective diameter $\sigma$, experience a wall corrugation of comparable size. Trapping effects resulting from such commensurability in length scales cannot be captured within a hydrodynamic description since the continuum approximation dictates that length scales characterizing the flow geometry and boundaries must be significantly larger than the fluid molecular size. The agreement between the MD and continuum solutions also worsens with increasing values of $k a$; the continuum results shown in 
figure 6(c) consistently overestimate the slip length with respect to the molecular-based simulations.

We investigate next if it is possible within a simplified statistical mechanical model to describe the behaviour of the fluid in the limit where the fluid particles are of comparable size with the wall inhomogeneities. This exercise helps reveal the molecular basis for liquid slip based on consideration of the friction coefficient characterizing the fluid/solid interface.

\section{Green-Kubo analysis of the friction coefficient for molecular scale wall corrugation}

In previous work (Bocquet \& Barrat 1994; Barrat \& Bocquet 1999a,b), it has been shown that the dependence of the slip length on molecular parameters can be computed from transport relations based on the fluctuation-dissipation theorem (Onsager 1931). This theorem relates the linear response of a system subject to a small external perturbation to the relaxation of spontaneous fluctuations in equilibrium. The approach, based on a Green-Kubo (GK) analysis (Heyes 1998), successfully predicts the shear viscosity, thermal conductivity and diffusivity of bulk liquids, by computing the relaxation profile of the auto-correlation functions associated with fluctuations in the shear stress, heat flux and velocity, respectively. Barrat \& Bocquet (1999a) used this method to relate the viscosity of a fluid subject to planar shear along the $\hat{x}$-axis to the dynamic friction coefficient $\beta$ (defined in $\S 1$ ) at the liquid/solid interface starting from the relation

$$
\beta \equiv \mu / L_{s}^{G K} \sim\left(A_{x y} k_{B} T\right)^{-1} \int_{0}^{\infty}\left\langle F_{x}(t) F_{x}(0)\right\rangle \mathrm{d} t .
$$

Here, $F_{x}(t)$ denotes the $x$-component of the force exerted by the wall on the liquid in equilibrium (i.e. in the absence of shear), the angular brackets $\langle\cdot\rangle$ denote the equilibrium ensemble average and $A_{x y}=L_{x} L_{y}$ is the liquid/solid interfacial area. The force auto-correlation function can be re-expressed in terms of the density autocorrelation function (DAF) (Barrat \& Bocquet 1999a):

$$
\mu / L_{s}^{G K} \sim\left(A_{x y} k_{B} T\right)^{-1} \int_{0}^{\infty} \mathrm{d} t \iint \mathrm{d} \boldsymbol{r}_{1} d \boldsymbol{r}_{2} F_{x}\left(\boldsymbol{r}_{1}\right) F_{x}\left(\boldsymbol{r}_{2}\right)\left\langle\rho\left(\boldsymbol{r}_{1}, t\right) \rho\left(\boldsymbol{r}_{2}, 0\right)\right\rangle,
$$

where $\boldsymbol{r}$ denotes the position of the liquid molecules.

Barrat \& Bocquet (1999a) approximated the force exerted by the wall lattice on the liquid by appealing to a simplified form of the interaction potential energy first derived by Steele (1973). Steele (1973) showed that the total energy of an isolated adsorbate gas atom interacting with the atoms in a crystalline solid of given lattice symmetry can be decomposed as a Fourier series in the position variables in the plane parallel to the surface (or equivalently, in multiples of the two-dimensional reciprocal lattice vectors $\boldsymbol{q}$ ) under the assumptions of pairwise additivity (gas atom-solid atom) and inverse power-law interactions. Barrat \& Bocquet (1999a) investigated the force exerted by the exposed (100) face of an FCC lattice by restricting the analysis to the top layer of wall atoms and only those atoms identified by the shortest reciprocal lattice vector $\boldsymbol{q}_{\|}$since these terms dominate the sum. Under these assumptions, a simplified potential

$$
V(x, y, z)=V_{o}(z)+V_{1}(z)\left[\cos \left(q_{\|} x\right)+\cos \left(q_{\|} y\right)\right]
$$


was used where $z$ denotes the vertical distance of the fluid particles from the wall atoms and $\boldsymbol{x}=(x, y)$ represents the position of fluid particles within the $(x, y)$-plane. For LJ interactions, the term $V_{o}(z)$, which corresponds to the $\boldsymbol{q}=0$ term in the Fourier series, scales as $0.4 \sigma_{w f}^{10} / z^{10}-\sigma_{w f}^{4} / z^{4} ; V_{1}(z)$ represents the additional contribution to the potential energy from the lateral arrangement of wall atoms within a plane which depends on lattice symmetry and relative size. The complete expression for $V(x, y, z)$, which incorporates the sum over all reciprocal lattice vectors and all layers comprising the solid wall, can be found in Steele (1973) for various lattice symmetries. Since $F_{x}=-\partial V / \partial x$, the approximate wall force is then given by $F_{x}(x, z)=q_{\|} V_{1}(z) \sin \left(q_{\|} x\right)$. Smith, Robbins \& Cieplak (1996) used a slightly different form of the wall potential for which $V_{1}(z) \rightarrow f V_{1}(z)$ so that the corrugation amplitude of the underlying lattice could be varied without modifying the adsorption energy $V_{o}(z)$. They investigated the dependence of the slip length on the parameter $f$ for $0.05 \lesssim f \lesssim 1.6$. Their analysis was conducted in support of friction experiments involving monolayer films of gas atoms deposited on substrates subject to lateral oscillation using a quartz crystal microbalance. One of the main conclusions of that study was that the slip time $t_{\text {slip }} \propto L_{s} \sim f^{-2}$.

In the current study, it is desirable to separate the effect of the intrinsic interaction between the wall atoms and liquid molecules from the influence of surface roughness. We therefore propose a simplified model potential of the form

$$
V(x, y, z)=V_{o}(z)+\frac{a}{\sigma} \tilde{V}_{1}(z) \cos (k x)+\frac{b}{\sigma} \tilde{V}_{1}(z)\left[\cos \left(q_{\|} x\right)+\cos \left(q_{\|} y\right)\right],
$$

where $k=2 \pi / \lambda$ is the wavenumber corresponding to a one-dimensional sinusoidal modulation of the wall surface. This model potential is not derived from Steele (1973) but represents only an approximate interaction potential which incorporates to first order the combined effects of fluid-wall interactions and substrate corrugation. The prefactor $a$ represents the corrugation amplitude in terms of the LJ length scale $\sigma ; b$ represents an effective embedding depth for the fluid particles within the solid lattice. The term $\tilde{V}_{1}(z)$ represents an effective scaling potential.

The $x$-component of the wall force is then given by

$$
F_{x}(x, z) \sim \frac{\tilde{V}_{1}(z)}{\sigma}\left[a k \sin (k x)+b q_{\|} \sin \left(q_{\|} x\right)\right],
$$

where the magnitude of the shortest reciprocal lattice vector corresponding to the simulations in $\S 2$ is given by $q_{\|}=4.52 \sigma^{-1}$. Upon substitution of (5.5) into (5.2), we find

$$
\begin{aligned}
\mu / L_{s}^{G K} \sim & \left(A_{x y} \sigma^{2} k_{B} T\right)^{-1} \\
& \times \int_{0}^{\infty} \mathrm{d} t \int \mathrm{d} x_{1} \mathrm{~d} y_{1} \mathrm{~d} z_{1} \int \mathrm{d} x_{2} \mathrm{~d} y_{2} \mathrm{~d} z_{2}\left\langle\rho\left(\boldsymbol{x}_{1}, z_{1}, t\right) \rho\left(\boldsymbol{x}_{2}, z_{2}, 0\right)\right\rangle \tilde{V}_{1}\left(z_{1}\right) \tilde{V}_{1}\left(z_{2}\right) \\
& \times\left\{q_{\|}^{2} b^{2} \sin \left(q_{\|} x_{1}\right) \sin \left(q_{\|} x_{2}\right)+k^{2} a^{2} \sin \left(k x_{1}\right) \sin \left(k x_{2}\right)\right. \\
& \left.+a b q_{\|} k\left[\sin \left(q_{\|} x_{1}\right) \sin \left(k x_{2}\right)+\sin \left(k x_{1}\right) \sin \left(q_{\|} x_{2}\right)\right]\right\}
\end{aligned}
$$

This equation can be simplified with reference to the Fourier transform of the fluid density in the plane of the substrate

$$
\rho_{\boldsymbol{q}}(z ; t)=\int \rho(\boldsymbol{x}, z ; t) \mathrm{e}^{\mathrm{i} \boldsymbol{q} \cdot \boldsymbol{x}} \mathrm{d} \boldsymbol{x},
$$


where $\boldsymbol{q}$ lies in the $(x, y)$-plane. Translational invariance of the density along the $\hat{x}$-axis (direction of shear) requires that $\left\langle\rho\left(x_{1}, t\right) \rho\left(x_{2}, 0\right)\right\rangle=\left\langle\rho\left(x_{1}-x_{2}, t\right) \rho(0,0)\right\rangle$, which leads to the following relation for the (complex) Fourier coefficients, namely $\left\langle\rho_{k}(t) \rho_{q}(0)\right\rangle \delta_{q,-k}=\left\langle\rho_{k}(t) \rho_{-k}(0)\right\rangle=\left\langle\rho_{k}(t) \rho_{k}^{*}(0)\right\rangle$. Equation (5.6) therefore reduces to

$$
\begin{aligned}
\mu / L_{s}^{G K} \sim & \int_{0}^{\infty} \mathrm{d} t \iint \mathrm{d} z_{1} \mathrm{~d} z_{2} \tilde{V}_{1}\left(z_{1}\right) \tilde{V}_{1}\left(z_{2}\right) \\
& \times\left[a^{2} k^{2}\left\langle\rho_{k}\left(z_{1}, t\right) \rho_{-k}\left(z_{2}, 0\right)\right\rangle+b^{2} q_{\|}^{2}\left\langle\rho_{q_{\|}}\left(z_{1}, t\right) \rho_{-q_{\|}}\left(z_{2}, 0\right)\right\rangle\right] .
\end{aligned}
$$

Barrat \& Bocquet (1999a) confirmed for the case of a flat wall that the DAF undergoes a diffusive-type relaxation according to

$$
\left\langle\rho_{q_{\|}}(t) \rho_{-q_{\|}}(0)\right\rangle=\exp \left(-D_{q_{\|}} q_{\|}{ }^{2} t\right)\left\langle\rho_{q_{\|}} \rho_{-q_{\|}}\right\rangle
$$

where $\left\langle\rho_{q_{\|}} \rho_{-q_{\|}}\right\rangle$is the static auto-correlation function and $D_{q_{\|}}$represents the in-plane diffusion coefficient describing the relaxation of density fluctuations within the first liquid layer. For a wall force given by (5.5), we find that the decay of the DAFs is instead described by stretched exponentials (Williams \& Watts 1970) of the form

$$
\left\langle\rho_{q_{\|}}\left(z_{1}, t\right) \rho_{-q_{\|}}\left(z_{2}, 0\right)\right\rangle=\exp \left[-\left(A_{q_{\|}} q_{\|}^{2} t\right)^{\beta_{q_{\|}}}\right]\left\langle\rho_{q_{\|}}\left(z_{1}\right) \rho_{-q_{\|}}\left(z_{2}\right)\right\rangle
$$

and

$$
\left\langle\rho_{k}\left(z_{1}, t\right) \rho_{-k}\left(z_{2}, 0\right)\right\rangle=\exp \left[-\left(A_{k} k^{2} t\right)^{\beta_{k}}\right]\left\langle\rho_{k}\left(z_{1}\right) \rho_{-k}\left(z_{2}\right)\right\rangle,
$$

where $A$ and $\beta$ represent fitting parameters determined from the MD simulations. Stretched exponentials often occur in systems subject to an intrinsic non-exponential relaxation or to a net relaxation caused by a spatially heterogeneous distribution of relaxation times. In our case, the non-exponential decay can be traced to the fact that the wavenumbers $q_{\|}$and $k$ are slightly displaced from the wavenumber (approximately $2 \pi / \sigma$ ) corresponding to the maximum peak in the in-plane liquid structure factor. The time integration in (5.8) then yields the coefficients proportional to each term in the sum (5.8), namely $\Gamma\left(1 / \beta_{q_{\|}}\right) /\left(\beta_{q_{\|}} A_{q_{\|}} q_{\|}{ }^{2}\right)$ and $\Gamma\left(1 / \beta_{k}\right) /\left(\beta_{k} A_{k} k^{2}\right)$ where $\Gamma$ denotes the Gamma function. The simulations yielded $A_{q_{\|}}=0.20 \pm 0.02 \sigma^{2} / \tau$, $A_{k}=0.48 \pm 0.02 \sigma^{2} / \tau, \beta\left(q_{\|}\right)=0.80 \pm 0.05$ and $\beta(k)=0.67 \pm 0.05$ for $k=3.45 \sigma^{-1}$, corresponding to the smallest-wavelength corrugation examined in $\S 4.2$.

In the MD simulations, the presence of a solid wall causes density oscillations in the adjacent liquid (not shown), with a maximum peak corresponding to the dense packing of liquid molecules in the first liquid layer. The dominant contribution to (5.8) can be estimated from the terms $z_{1} \approx z_{2}$, where $z_{1}$ from hereon denotes the $z$-coordinate of the peak in the density profile $\rho(z)$. Under this assumption, the DAF can be re-expressed in terms of the structure factor characterizing the first liquid layer where $\left\langle\rho_{q_{\|}}\left(z_{1}\right) \rho_{-q_{\|}}\left(z_{2}\right)\right\rangle \approx A_{x y}\left\langle\rho\left(z_{1}\right)\right\rangle \delta\left(z_{1}-z_{2}\right) S_{1}\left(q_{\|}\right)$and

$$
S_{1}\left(q_{\|}\right)=\frac{1}{A_{x y}\left\langle\rho\left(z_{1}\right)\right\rangle}\left|\sum_{i} \mathrm{e}^{\mathrm{i} q_{\|} x_{i}}\right|^{2} .
$$

The factor $A_{x y}\left\langle\rho\left(z_{1}\right)\right\rangle$ normalizes the summation by the number of fluid particles in the first layer. This simplification yields

$$
\mu / L_{s}^{G K} \sim \frac{\rho_{c}}{\sigma^{2} k_{B} T} \int \mathrm{d} z \tilde{V}_{1}^{2}(z)\left[b^{2} S_{1}\left(q_{\|}\right) / D_{q_{\|}}+a^{2} S_{1}(k) / D_{k}\right],
$$

where the effective diffusion coefficients are given by $D_{\alpha}=A_{\alpha} \beta_{\alpha} / \Gamma\left(1 / \beta_{\alpha}\right)$ for $\alpha=q_{\|}, k$ and the contact density $\rho_{c}=\rho\left(z_{1}\right)$. The final relation for the effective slip length is 


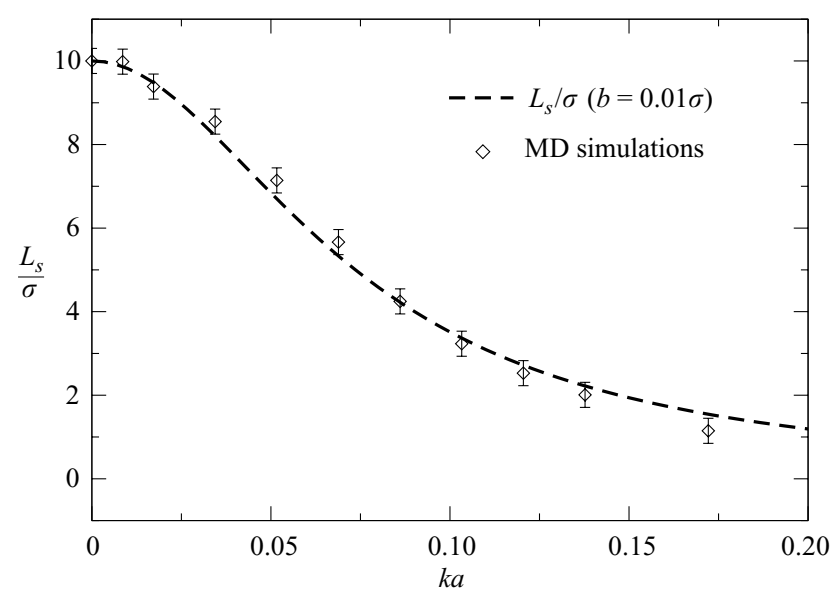

FIGURE 7. Comparison of the analytic result (5.14) (dashed line) obtained from the simplified Green-Kubo analysis with results of MD simulations (diamonds - same data as in figure 6) for $0 \leqslant k a \leqslant 0.2$ where $\lambda=2 \pi / k=1.82 \sigma$. The only fitting parameter is $b=0.01 \sigma$.

then given by

$$
\frac{L_{s}^{G K}(a)}{L_{s}(a=0)}=\left[1+\frac{a^{2}}{b^{2}} \frac{S_{1}(k)}{D_{k}} \frac{D_{q_{\|}}}{S_{1}\left(q_{\|}\right)}\right]^{-1} .
$$

where the intrinsic slip length $L_{s}(a=0) \propto D_{q_{\|}} /\left[b^{2} S_{1}\left(q_{\|}\right)\right]$. As is evident, an increase in the wall modulation amplitude $a$ leads to a decrease in the effective slip length which scales as $a^{-2}$. The same scaling has been reported by Smith et al. (1996) in studies of isolated gas atoms interacting with a crystalline lattice.

Figure 7 is a direct comparison of the slip length as computed from the velocity profiles in the MD simulations (same data as in figure 6) with the analytic prediction (5.14) for $\lambda=1.82 \sigma$ where the only fitting parameter is $b=0.01 \sigma$. As in $\S 4.2$, the slip length corresponding to a flat wall was set to $L_{s}(a=0)=(10.0 \pm 0.3) \sigma$. The quantities in (5.14) were computed from equilibrium MD simulations where $S_{1}\left(q_{\|}\right)=\lim _{t \rightarrow 0}\left(N_{1}\right)^{-1}\left\langle\rho_{q_{\|}}(t) \rho_{-q_{\|}}(0)\right\rangle$ and $N_{1}$ is the average number of particles in the first liquid layer. Within statistical errors, the ratio $S(k) D_{q_{\|}} / S\left(q_{\|}\right) D_{k}=0.20 \pm 0.02$ was found to be independent of $a$ for $0 \leqslant k a \leqslant 0.2$. The agreement between the MD results and (5.14) is rather good throughout the range $0 \leqslant k a \leqslant 0.15$, or equivalently for amplitudes $a \leqslant 0.043 \sigma$. The molecular simulation data fall systematically below the dashed curve beyond this range. For $k a=0.3$ (i.e. $a=0.086 \sigma$ ), the no-slip condition is recovered; as $k a \rightarrow 1, L_{s} \rightarrow-1.0 \sigma$ indicative of a trapped layer of liquid adjacent to the wall.

The excellent correspondence between the MD simulations and this simplified model for $k a \lesssim 0.15$ (i.e. in the limit of molecular-scale roughness) suggests that the friction coefficient can be simply viewed as the summation of two independent sources of resistance to flow, one from the LJ interactions between the liquid and a flat wall and the other from the potential describing small-scale topological features or surface roughness. This description is only valid, however, for sufficiently small amplitudes and wavelengths close to $\sigma$, the characteristic length scale established by the LJ interactions. The MD simulations show that disturbances from the wall modulation distort the flow field within a distance $\lambda$ of the wall. Corrugation wavelengths larger 
than molecular-scale distortions therefore perturb the liquid well beyond the first layer thereby violating the assumptions leading to (5.14).

\section{Conclusions}

The slip length characterizing the surface of contact between a liquid and solid in relative motion is influenced by a number of factors. These include the intrinsic affinity and commensurability between the liquid and solid molecular size as well as the surface roughness caused by imperfections and other heterogeneities. In this paper, we investigate the effects of periodic roughness by modelling the bounding surface as a sinusoid of amplitude $a$ and wavenumber $k=2 \pi / \lambda$ and examine the behaviour of the slip length as a function of $k a$. We restrict this study to Newtonian liquids subject to steady planar shear where in the MD simulations $R e \lesssim 10$ and in the continuum solutions $R e=0$. The solutions obtained from MD simulations are directly compared to the Stokes flow solutions as well as a simplified statistical mechanical model for the friction coefficient between the wall and first liquid layer. In all cases examined, the slip length decreases monotonically with increasing values of $k a$. A no-slip or even negative-slip boundary condition can be obtained for sufficiently large values of $k a$. In general, the smaller the corrugation wavelength, the smaller the corrugation amplitude required to reproduce such immobilization conditions.

The MD results recover the continuum solutions in the Stokes regime in the limit of small $k a$ and large $\lambda$. This agreement demonstrates that molecular-based simulations can successfully reproduce hydrodynamic predictions provided that the length scales characterizing substrate inhomogeneities are large compared to the liquid molecular diameter. This restriction forms the basis of the continuum approximation underlying the hydrodynamic equations of motion. As the substrate wavelength approaches length scales comparable with the liquid molecular diameter, the continuum solutions overestimate the degree of slip. In this case, the continuum analysis is unable to capture the effects associated with fluid particle trapping by molecular-scale wall corrugations.

In the limit where the corrugation wavelength approaches the liquid molecular diameter, we develop a simplified model of the friction coefficient characterizing the liquid/solid interface based on an earlier study of Barrat \& Bocquet (1999a). The friction coefficient is inversely proportional to the slip length. The slip length, which effectively represents the linear response of the system to flow at low shear rates, is shown to be proportional to the decay rate of spontaneous equilibrium density fluctuations in the presence of a wavy wall. The potential energy exerted by the wavy wall exhibits two competing wavelengths, one set by the lattice sites comprising a flat crystalline wall and the other set by the topological modulation describing a periodically roughened wall. A direct comparison between the resulting analytic relation for the effective slip length and the MD results shows excellent agreement for the smallest wavelength studied, namely $\lambda=1.82 \sigma$.

Despite the success in providing an analytic description for the MD results for small- and large-wavelength modulation, there is interest in designing algorithms that can smoothly span length scales from the molecular to macroscopic dimensions. Hybrid algorithms have been proposed which can successfully stitch together the results of MD simulations with large-scale continuum solvers. O'Connell \& Thompson (1995) were the first to develop a hybrid approach based on constrained dynamics in an overlap region bridging the particle (MD) and continuum solutions. This technique was applied to the transient startup problem for plane Couette flow 
where the overlap regime is oriented parallel to the solid boundaries. Subsequent studies by a number of groups have refined and extended this approach to problems involving multiple time scales and more complex flows (Hadjiconstantinou \& Patera 1997; Li, Liao \& Yip 1998; Flekkoy, Wagner \& Feder 2000; Nie et al. 2004; Ren \& E 2005) including rather difficult systems involving the flow of water in the vicinity of carbon nanotubes (see the review by Koumoutsakos (2005) and references therein). These approaches have proved extremely successful in reproducing flow characteristics spanning multiple scales and will probably become a mainstay of hydrodynamic simulations. It would be interesting next to develop such hybrid approaches for investigating both shear and pressure-driven flow along periodically roughened and randomly roughened surfaces.

This work is supported by grants from the NASA Microgravity Fluid Physics program, the National Science Foundation - Sensor and Sensor Networks Program, and the US Army TACOM-ARDEC. S.M.T. also gratefully acknowledges the hospitality and financial support of the Moore Distinguished Scholar Program at the California Institute of Technology where this work was completed, as well as the Princeton University Computing Facility for use of the Beowulf cluster. Dr A. A. Darhuber kindly provided the data in figure 6(c).

\section{REFERENCES}

Allen, M. P. \& Tildesley, D. J. 1987 Computer Simulation of Liquids. Clarendon.

BARRAT, J.-L. \& BOCQUET, L. 1999a Influence of wetting properties on hydrodynamic boundary conditions at a fluid/solid interface. Faraday Discuss. 112, 119.

Barrat, J.-L. \& Bocquet, L. $1999 b$ Large slip effect at a nonwetting fluid-solid interface. Phys. Rev. Lett. 82, 4671.

Baudry, J., Charlaix, E., Tonck, A. \& Mazuyer, D. 2001 Experimental evidence for a large slip effect at a nonwetting fluid-solid interface. Langmuir 17, 5232.

Bocquet, L. \& BARRAT, J.-L. 1994 Hydrodynamic boundary conditions, correlation functions, and kubo relations for confined fluids. Phys. Rev. E 49, 3079.

Choi, C. H., Westin, K. J. A. \& Breuer, K. S. 2003 Apparent slip flows in hydrophilic and hydrophobic microchannels. Phys. Fluids 15, 2897.

Cottin-Bizonne, C., Cross, B., Steinberger, A. \& Charlaix, E. 2005 Boundary slip on smooth hydrophobic surfaces: Intrinsic effects and possible artifacts. Phys. Rev. Lett. 94, 056102.

Darhuber, A. A., Priezjev, N. V. \& Troian, S. M. 2006 Slip length behavior in Poiseuille flow bounded by roughened walls: continuum predictions versus molecular dynamics simulations. Phys. Rev. E. (to be submitted).

Dussan V., E. B. 1979 Spreading of liquids on solid-surfaces: static and dynamic contact lines. Annu. Rev. Fluid Mech. 11, 371.

Dussan V., E. B. \& Davis, S. H. 1974 Motion of a fluid-fluid interface along a solid-surface. J. Fluid Mech. 65, 71.

Einzel, D., PANZER, P. \& LiU, M. 1990 Boundary-condition for fluid flow - curved or rough surfaces. Phys. Rev. Lett. 64, 2269.

Einzel, D. \& Parpia, J. M. 1997 Slip in quantum fluids. J. Low Temp. Phys. 109, 1.

Flekkoy, E. G., Wagner, G. \& Feder, J. 2000 Hybrid model for combined particle and continuum dynamics. Europhys. Lett. 52, 271.

Galea, T. M. \& ATtaRd, P. 2004 Molecular dynamics study of the effect of atomic roughness on the slip length at the fluid-solid boundary during shear flow. Langmuir 20, 3477.

GaO, J. P., LuedtKe, W. D. \& Landman, U. 1997 Layering transitions and dynamics of confined liquid films. Phys. Rev. Lett. 79, 705.

GAO, J. P., LuedtKe, W. D. \& LANDMAn, U. 2000 Structures, solvation forces and shear of molecular films in a rough nano-confinement. Tribol. Lett. 9, 3. 
Goldstern, S. (Ed.) 1938 Modern Developments in Fluid Dynamics: An Account of Theory and Experiment Relating to Boundary Layers Turbulent Motion and Wakes. Oxford University Press.

Greenspan, H. 1978 Motion of a small viscous droplet that wets the surface. J. Fluid Mech. 84, 125.

Grest, G. S. \& Kremer, K. 1986 Molecular dynamics simulation for polymers in the presence of a heat bath. Phys. Rev. A 33, 3628.

van Gunsteren, W. F., Berendsen, H. J. C. \& Rullmann, J. A. C. 1981 Stochastic dynamics for molecules with constraints: Brownian dynamics of normal alkanes. Mol. Phys. 44, 69.

Hadjiconstantinou, N. G. \& Patera, A. T. 1997 Heterogeneous atomistic-continuum representations for dense fluid systems. Intl J. Mod. Phys. 8, 967.

HeYes, D. M. 1998 The Liquid State: Applications of Molecular simulations. J. Wiley \& Sons.

Hocking, L. M. 1976 A moving fluid interface on a rough surface. J. Fluid Mech. 76, 801.

Horn, R. G., Vinogradova, O. I., Mackay, M. E. \& Phan-Thien, N. 2000 Hydrodynamic slippage inferred from thin film drainage measurements in a solution of nonadsorbing polymer. J. Chem. Phys. 112, 6424.

Huh, C. \& Scriven, L. E. 1971 Hydrodynamic model of steady movement of a solid/liquid/fluid contact line. J. Colloid Interface Sci. 35 (1), 85.

Ishida, N., Inoue, T., Miyahara, M. \& Higashitani, K. 2000 Nano bubbles on a hydrophobic surface in water observed by tapping mode atomic force microscopy. Langmuir 16, 6377.

Jabbarzadeh, A., Atkinson, J. D. \& Tanner, R. I. 2000 Effect of the wall roughness on slip and rheological properties of hexadecane in molecular dynamics simulation of Couette shear flow between two sinusoidal walls. Phys. Rev. E 61, 690.

JANSONS, K. M. 1988 Determination of the macroscopic (partial) slip boundary condition for a viscous flow over a randomly rough surface with a perfect slip microscopic boundary condition. Phys. Fluids 31, 15.

Jin, S., Huang, P., Park, J., Yoo, J. Y. \& Breuer, K. S. 2004 Near-surface velocimetry using evanescent wave illumination. Exps. Fluids 37, 825.

Koplik, J. \& Banavar, J. R. 1995 Continuum deductions from molecular hydrodynamics. Annu. Rev. Fluid Mech. 27, 257.

Koumoutsakos, P. 2005 Multiscale flow simulations using particles. Annu. Rev. Fluid. Mech. 37, 457.

LI, J., LiaO, D. \& YIP, S. 1998 Coupling continuum to molecular-dynamics simulation: reflecting particle method and the field estimator. Phys. Rev. E 57, 7259.

lumma, D., Best, A., Gansen, A., Feuillebois, F., Radler, J. O. \& Vinogradova, O. I. 2003 Flow profile near a wall measured by double-focus fluorescence cross-correlation. Phys. Rev. E 67, 056313.

Migler, K. B., Hervet, H. \& Leger, L. 1993 Slip transition of a polymer melt under shear stress. Phys. Rev. Lett. 70, 287.

Miksis, M. J. \& Davis, S. H. 1994 Slip over rough and coated surfaces. J. Fluid Mech. 273, 125.

Moffatt, H. K. 1964 Viscous and resistive eddies near a sharp corner. J. Fluid Mech. 18, 1.

Nie, X. B., Chen, S. Y., E, W. \& Robbins, M. O. 2004 A continuum and molecular dynamics hybrid method for micro- and nano-fluid flow. J. Fluid Mech. 500, 55.

O'Connell, S. T. \& Thompson, P. A. 1995 Molecular dynamics-continuum hybrid computations: a tool for studying complex flows. Phys. Rev. E 52, R5792.

Onsager, L. 1931 Reciprocal relations in irreversible processes. ii. Phys. Rev 38, 2265.

Panzer, P., Liu, M. \& Einzel, D. 1992 The effects of boundary curvature on hydrodynamic fluid flow - calculation of slip lengths. Intl J. Mod. Phys. B 6 (20), 3251.

Pit, R., Hervet, H. \& Leger, L. 2000 Direct experimental evidence of slip in hexadecane: Solid interfaces. Phys. Rev. Lett. 85, 980.

Priezjev, N. V., Darhuber, A. A. \& Troian, S. M. 2005 Slip behaviour in liquid films on surfaces of patterned wettability: Comparison between continuum and molecular dynamics simulations. Phys. Rev. E 71, 041608.

Priezjev, N. V. \& Troian, S. M. 2004 Molecular origin and dynamic behaviour of slip in sheared polymer films. Phys. Rev. Lett. 92, 018302.

REN, W. Q. \& E, W. 2005 Heterogeneous multiscale method for the modeling of complex fluids and micro-fluidics. J. Comput. Phys. 204, 1.

Richardson, S. 1973 On the no-slip boundary condition. J. Fluid Mech. 59, 707. 
Schmatko, T., Hervet, H. \& Leger, L. 2005 Friction and slip at simple fluid-solid interfaces: the roles of the molecular shape and the solid-liquid interaction. Phys. Rev. Lett. 94, 244501.

Smith, E. D., Robbins, M. O. \& Cieplak, M. 1996 Friction on adsorbed monolayers. Phys. Rev. B 54, 8252 .

SteELE, W. A. 1973 Physical interaction of gases with crystalline solids. 1. gas-solid energies and properties of isolated adsorbed atoms. Surface Sci. 36, 317.

Steitz, R., Gutberlet, T., Hauss, T., Klosgen, B., Krastev, R., Schemmel, S., Simonsen, A. C. \& FINDENEGG, G. H. 2003 Nanobubbles and their precursor layer at the interface of water against a hydrophobic substrate. Langmuir 19, 2409.

Stevens, M. J., Mondello, M., Grest, G. S., Cui, S. T., Cochran, H. D. \& Cummings, P. 1997 Comparison of shear flow of hexadecane in a confined geometry and in bulk. J. Chem. Phys. 106, 7303.

Thompson, P. A., Brinckerhoff, W. B. \& Robbins, M. O. 1993 Microscopic studies of static and dynamic contact angles. J. Adhesion Sci. Technol. 7, 535.

Thompson, P. A. \& Robbins, M. O. 1989 Simulations of contact line motion: slip and the dynamic contact angle. Phys. Rev. Lett. 63, 766.

Thompson, P. A. \& Robisns, M. O. $1990 a$ Origin of stick-slip motion in boundary lubrication. Science 250, 792.

Thompson, P. A. \& Robbins, M. O. $1990 b$ Shear flow near solids - epitaxial order and flow boundary conditions. Phys. Rev. A 41, 6830.

Thompson, P. A., Robbins, M. O. \& Grest, G. S. 1995 Structure and shear response in nanometer thick films. Israel J. Chem. 35, 93.

Thompson, P. A. \& Troian, S. M. 1997 A general boundary condition for liquid flow at solid surfaces. Nature 389, 360.

Tretheway, D. C. \& Meinhart, C. D. 2002 Apparent fluid slip at hydrophobic microchannel walls. Phys. Fluids 14, L9.

Tuck, E. O. \& Kouzoubov, A. 1995 A laminar roughness boundary condition. J. Fluid Mech. 300, 59.

Tyrrell, J. W. G. \& AtTard, P. 2001 Images of nanobubbles on hydrophobic surfaces and their interactions. Phys. Rev. Lett. 87, 176104.

Williams, G. \& WatTs, D. C. 1970 Non-symmetrical dielectric relaxation behaviour arising from a simple empirical decay function. Trans. Faraday Soc. 66, 80.

Zhu, Y. X. \& Granick, S. 2002 Limits of the hydrodynamic no-slip boundary condition. Phys. Rev. Lett. 88, 106102. 